\title{
IMPROVEMENTS IN BIASING AND COMPENSATION OF CMOS OPAMPS
}

\author{
Sean Nicolson and Khoman Phang \\ \{nicolson,kphang\}@eecg.utoronto.ca \\ The Edward S. Rogers Sr. Department of Electrical and Computer Engineering, University of Toronto \\ 10 King's College Road, Toronto, Ontario, M5S 3G4, Canada
}

\begin{abstract}
In this paper, we present modifications to the constant-gm bias circuit and the Miller-lead compensation technique which eliminate or minimize some of their shortcomings. First, we demonstrate how parasitic pad capacitance can cause instability in the constant-gm bias circuit, and show that the transconductance is constant only for specific bias conditions. Next, we suggest a new circuit topology that requires $75 \%$ less compensation capacitance to achieve stability. We also discuss problems with Miller-lead compensation that arise from temperature, process, and load variations. Finally, we present a new biasing technique to correct these problems, and, through simulation, demonstrate a $40^{\circ}$ improvement in phase margin over load current variations.
\end{abstract}

\section{INTRODUCTION}

Operational amplifiers are typically biased and compensated to minimize temperature and process variations in their open loop response. Although constant-gm biasing and Miller-lead compensation remain useful for this purpose, the sensitivity of these techniques can be improved over temperature, process, and load variations.

To understand how the open loop characteristics of an opamp can vary, consider the opamp in Figure 1.

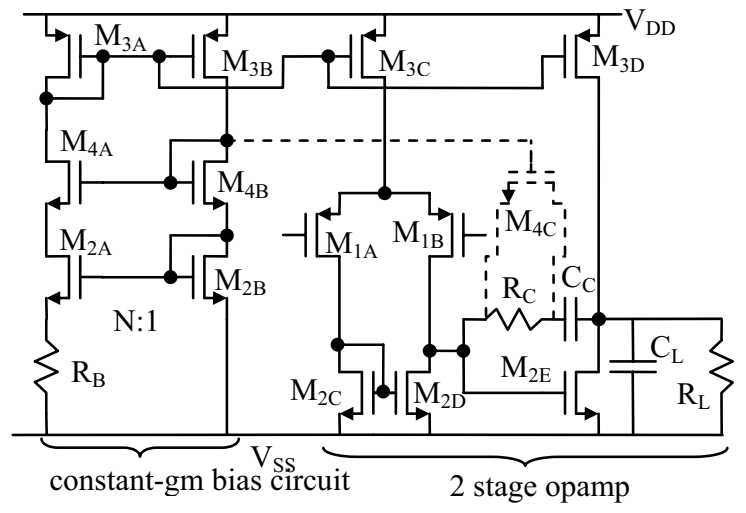

Figure 1: A 2 stage opamp with constant-gm biasing. In all figures, devices with the same numeric index are matched devices.

The pole and zero locations of the open loop response are given by (1)-(3), where $R_{C}$ is either a passive resistor, or implemented using the channel resistance of $M_{4 C}$ operating in the triode region [1]. Typically, the opamp is designed such that $f_{T}<f_{Z}<f_{2}$. Assuming dominant pole behavior, $f_{T}$ is given by (4).

$$
\begin{gathered}
f_{1}=\frac{1}{2 \pi g m_{2 E} C_{C} R_{O 1} R_{O 2}} \text { (1) } f_{2}=\frac{-g m_{2 E}}{2 \pi\left(C_{C}+C_{L}\right)} \\
f_{Z} \approx \frac{-1}{2 \pi R_{C} C_{C}} \quad \text { (3) } \\
f_{T} \approx \frac{g m_{1}}{2 \pi C_{C}}
\end{gathered}
$$

Clearly, changes in $g m_{1}, g m_{2}, R_{C}$, and $I_{D 2 E}$ caused by process, temperature, and load variations will affect the relationship between $f_{T}, f_{Z}$, and $f_{2}$. In contrast, an ideal biasing scheme would render $f_{1}, f_{2}, f_{Z}$, and $f_{T}$ constant.

The constant-gm bias circuit and the use of a MOSFET $R_{C}$ for compensation have numerous disadvantages. In Section II, we show that the constant-gm bias circuit does not always keep gm constant, and indicate exactly how variations in $g m$ arise. We also demonstrate that the constant-gm bias circuit can be unstable. In Section III, we introduce a new constant-gm bias circuit with improved stability. Section IV discusses disadvantages of the MOSFET implementation of $R_{C}$ shown in Figure 1, and introduces an improved technique for biasing $M_{4 C}$ for compensation.

\section{LIMITATIONS IN TRACKING GM}

Ideally, the constant-gm bias circuit in Figure 1 forces all MOSFETs (n/p-channel) to have constant $\mathrm{gm}$ over process and temperature [1,2]. Unfortunately, the application of constant-gm biasing to CMOS opamps is complicated by the MOSFET I-V characteristics. While one equation governs the $\mathrm{I}-\mathrm{V}$ characteristics of the BJT for the entire active region, the $\mathrm{I}-\mathrm{V}$ characteristics of the MOSFET change from weak to strong inversion. In this section, we investigate variations in gm caused when a constant-gm bias circuit with MOSFETs operating in a particular region of operation is used to bias other MOSFETs in either the same, or a different, region of operation.

\section{Case A. Strong Inversion Only}

Traditionally, all MOSFETs were biased in strong inversion where the $I_{D^{-}} V_{G S}$ relationship was governed by the square law. In such a case, consider any MOSFET $M_{X}$ biased by the constant-gm circuit in Figure 1. The drain current of $M_{2 B}$ $\left(I_{D 2 B}\right)$ and $g m_{X}$ are given by (5) and (6) respectively, where $\mu_{X}$ is either $\mu_{n}$ or $\mu_{p}$ depending on the device type. Substituting (5) into (6) yields (7), an expression for $g m_{X}$. If $M_{X}$ and $M_{2 B}$ 
are both n-channel MOSFETs, then (7) is independent of process and temperature. On the other hand, if $M_{X}$ is a pchannel MOSFET, then the final term in (7) $\left(\mu_{p} / \mu_{n}\right)$ varies extensively with process and slightly with temperature.

$$
\begin{gathered}
I_{D 2 B}=\frac{1}{R_{B}^{2}}\left(1-\frac{1}{\sqrt{N}}\right)\left(\frac{2}{\mu_{n} C_{O X}(W / L)_{2 B}}\right) \\
g m_{X}=\sqrt{2 \mu_{n} C_{O X}(W / L)_{X} I_{D X}} \\
g m_{X}=\sqrt{\frac{2}{R_{B}^{2}}\left(1-\frac{1}{\sqrt{N}}\right)\left(\frac{(W / L)_{X}}{(W / L)_{2 B}}\right)\left(\frac{\mu_{X}}{\mu_{n}}\right)}
\end{gathered}
$$

Case B. Strong Inversion $\left(M_{2 B}\right)$ and Weak Inversion $\left(M_{X}\right)$ Consider the case where $M_{X}$ is in weak inversion and $M_{2 B}$ is in strong inversion. In weak inversion, $g m_{X}$ is given by (8), where $\phi_{t}$ is the thermal voltage, and $n$ is the slope factor $(n \approx$ 1.2) [3]

$$
g m_{X}=\frac{I_{D X}}{n \phi_{t}}
$$

Substituting the original expression for $I_{D 2 B}$ (and therefore $I_{D X}$ ) into (8), yields (9) which again depends on temperature $\left(\phi_{t}\right.$, $\left.\mu_{n}\right)$ and process $\left(\mu_{n}\right)$.

$$
g m_{X}=\frac{1}{n \phi_{t} R_{B}^{2}}\left(1-\frac{1}{\sqrt{N}}\right)\left(\frac{2}{\mu_{n} C_{O X}(W / L)_{2 B}}\right)
$$

\section{Case C. Weak Inversion Only}

If $M_{2 B}$ is also in weak inversion, the expression for $I_{D 2 B}$ (and therefore $I_{D X}$ ) is given by (10), and the resulting expression for $g m_{X}$ given by (11). Clearly, $g m_{X}$ is constant once again, with an advantage over strong inversion (case A): the ratio $\mu_{X} / \mu_{n}$ is absent from the expression for $g m_{X}$. Therefore, $g m_{X}$ is independent of process and temperature, even if $M_{X}$ and $M_{2 B}$ are different types.

$$
\begin{aligned}
& I_{D 2 B}=\frac{n \phi_{t}}{R_{B}} \ln (N) \\
& g m_{X}=\frac{1}{R_{B}} \ln (N)
\end{aligned}
$$

Case D. Weak Inversion $\left(M_{2 B}\right)$ and Strong Inversion $\left(M_{X}\right)$ When $M_{2 B}$ is in weak inversion and $M_{X}$ is in strong inversion, $g m_{X}$ is given by (12).

$$
g m_{X}=\sqrt{2 \mu_{n} C_{O X}(W / L)_{X} \frac{n \phi_{t}}{R_{B}} \ln (N)}
$$

Once again, $g m_{X}$ varies with process and temperature. However, on account of the square root dependence of $\mathrm{gm}$ on $I_{D}$ in strong inversion, the variations in (12) are less than in (10). Note that if $R_{B}$ is integrated on chip, process variations in $R_{B}$ influence (12) less than (7), (9), or (11).

In addition to our analysis, we simulated cases A-D in a $0.8 \mu \mathrm{m}$ BiCMOS process using HSPICE. In our simulations, all MOSFETs had identical drain currents regardless of their region of operation. Furthermore, all MOSFETs in a given region of operation had the same $V_{G S}-V_{T 0}$, regardless of their type ( $\mathrm{p}$ or $\mathrm{n}$ channel). We swept the simulator temperature from $0^{\circ} \mathrm{C}$ to $50^{\circ} \mathrm{C}$, and used corner models to simulate process variations. Table 1 shows our results. Except where indicated, $M_{2 B}$ and $M_{X}$ in Table 1 are n-channel MOSFETs.

Table 1. Process and temperature variations in the gm of MOSFETs biased with a constant-gm bias circuit.

\begin{tabular}{|l|l|l|c|c|}
\hline Case & \multicolumn{1}{|c|}{$\begin{array}{c}M_{2 B} \\
(\mathrm{n} \text {-channel) }\end{array}$} & \multicolumn{1}{|c|}{$M_{X}$} & $\begin{array}{c}\text { Temp } \\
\left(0^{\circ} \mathrm{C}-50^{\circ} \mathrm{C}\right)\end{array}$ & Process \\
\hline A & str. inv. & str. inv. & $0.5 \%$ & $0.1 \%$ \\
\hline A & str. inv. & $\begin{array}{l}\text { str. inv. } \\
\text { (p-channel) }\end{array}$ & $1 \%$ & $13 \%$ \\
\hline B & str. inv. & weak inv. & $8 \%$ & $7 \%$ \\
\hline C & weak inv. & weak inv. & $0.2 \%$ & $1 \%$ \\
\hline C & weak. inv. & $\begin{array}{l}\text { weak inv. } \\
\text { (p-channel) }\end{array}$ & $0.2 \%$ & $5 \%$ \\
\hline D & weak inv. & str. inv. & $5 \%$ & $4 \%$ \\
\hline
\end{tabular}

Based on our analysis and simulations we can draw several conclusions:

- $M_{X}$ and $M_{2 B}$ must always have the same bias point (same $V_{G S}-V_{T 0}$ ) to keep $g m_{X}$ constant over temperature and/or process [4].

- For MOSFETs in strong inversion, $M_{X}$ and $M_{2 B}$ must be the same type (n or p-channel) to obtain constant gm over temperature and process.

- To obtain only temperature independence of $g m_{X}$ in strong inversion, $M_{X}$ and $M_{2 B}$ may be different types, provided that the ratio $\mu_{X} / \mu_{n}$ does not vary significantly with temperature.

- For MOSFETs in weak inversion (and BJTs in general), temperature and process independence may be achieved even when $M_{X}$ and $M_{2 B}$ are different types.

\section{STABILITY IN CONSTANT-GM BIASING}

Another problem with the constant-gm bias circuit is that it can oscillate if not designed correctly. Typically, $R_{B}$ is implemented off-chip, and therefore a capacitance of several $\mathrm{pF}$ may be present in parallel with $R_{B}$, as shown in Figure 2 [5].

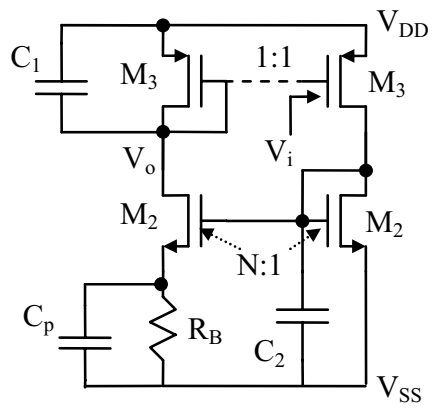

Figure 2: A simplified schematic of the constant-gm bias circuit, useful for small signal analysis. 
Consider breaking the feedback loop between the gates of $M_{3 A}$ and $M_{3 B}$, and applying an input signal at the gate of $M_{3 B}$. Using small signal analysis, $V_{o} / V_{i}$ is given by (13). Note that the loop gain from $V_{i}$ to $V_{o}$ is positive.

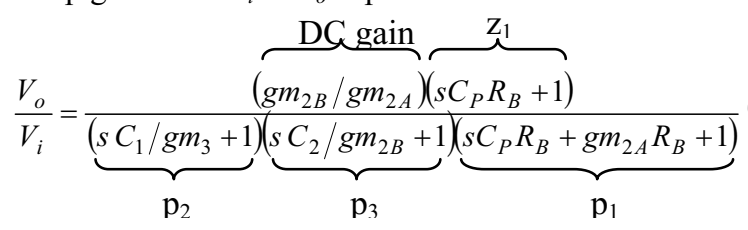

Typically, parasitic capacitances $C_{I}$ and $C_{2}$ are very small compared to $C_{P}$. In this case, $p_{1}$ and $z_{l}$ form a dominant polezero doublet, where the zero is always lower than the pole. If $g m_{2 A}$ is too large, then $z_{l}$ and $p_{I}$ move further apart, and $\left|V_{o} / V_{i}\right|$ can become greater than $0 \mathrm{~dB}$, as shown in Figure 3. When $\left|V_{o} / V_{i}\right|>0 \mathrm{~dB}$, the phase lag introduced by $p_{2}$ and $p_{3}$ can cause instability.

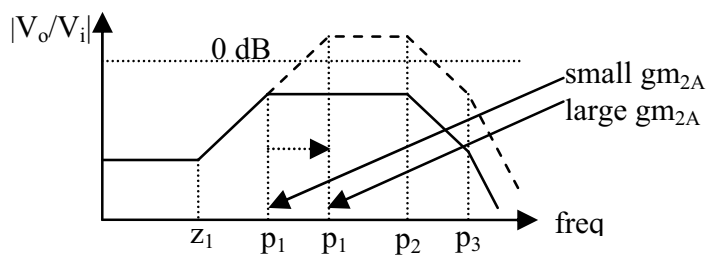

Figure 3: Magnitude plots for the constant-gm open loop function in (13)

To ensure stability, a compensation capacitor can be added to increase either $C_{1}$ or $C_{2}$, thereby creating a pole dominant over $p_{1}$. Unfortunately, $p_{2}$ and $p_{3}$ are formed by low impedance nodes, necessitating a compensation capacitance on the order of $C_{P}$. For example, to ensure stability with $C_{2}$, inequality (14) must be satisfied.

$$
C_{2}>\left(\frac{g m_{2 A} R_{B}}{g m_{2 A} R_{B}+1}\right) C_{P} \approx C_{P}
$$

\section{A NEW CONSTANT-GM BIAS CIRCUIT}

Modifying the constant-gm circuit in Figure 2 to include a high impedance node reduces the size of the compensation capacitor needed to ensure stability. Shown in Figure 4 is the modified constant-gm circuit, which was originally presented in [5].

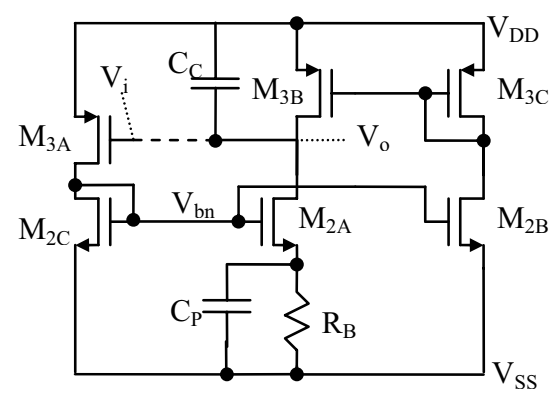

Figure 4: A new constant-gm bias circuit with a high impedance node.
MOSFETs $M_{3 B}, M_{3 C}, M_{2 A}$, and $M_{2 B}$ form the original constant$g m$ circuit. However, the addition of $M_{3 A}$ and $M_{2 C}$ makes $V_{o}$ a high impedance node. A compensation capacitor $\left(C_{C}\right)$ is placed at the high impedance node. Ignoring the degeneration of $\mathrm{ro}_{2 A}$, the open loop transfer function of the new constantgm circuit is approximately given by (15).

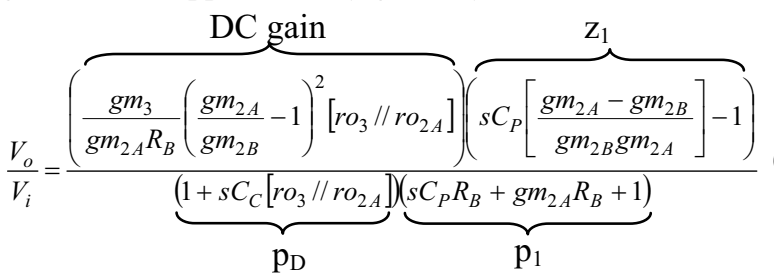

The new transfer function contains the original pole formed by $C_{P}$ and $R_{B}$, but also has a new dominant pole formed by $C_{C}$ and $\mathrm{ro}_{2 B} / / \mathrm{ro}_{3}$. Alternatively, $C_{C}$ can be connected between $V_{o}$ and $V_{b n}$ instead of between $V_{o}$ and $V_{D D}$. Connecting $C_{C}$ to $V_{b n}$ takes advantage of the Miller effect introduced by the voltage gain from $V_{o}$ to $V_{b n}$. If necessary, a resistor can be added in series with $C_{C}$ to introduce lead compensation.

To demonstrate the improved stability of the proposed constant-gm bias circuit, we simulated the circuits in Figure 2 and Figure 4 with $C_{2}=4 \mathrm{pF}$ and $C_{C}=1 \mathrm{pF}$ compensation capacitors, respectively. We designed both circuits to have the same bias currents and transistor sizes. As shown in Figure 5, the proposed circuit is stable, despite its smaller compensation capacitor.

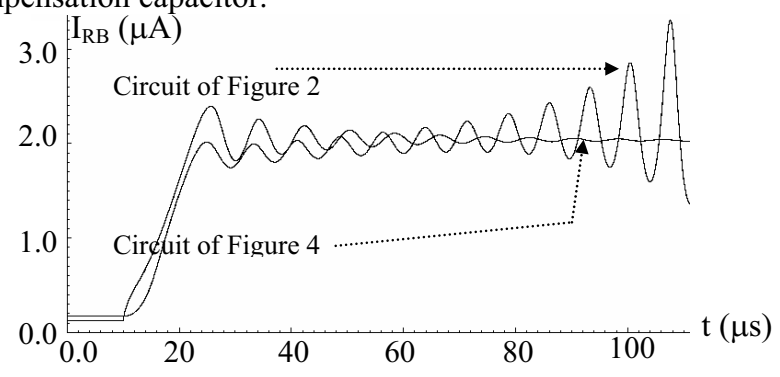

Figure 5: Start-up simulations showing instability in the traditional constant-gm bias circuit. $\mathrm{C}_{\mathrm{P}}=40 \mathrm{pF}$ in both cases.

\section{A GM-TRACKING LEAD COMPENSATION BIASING TECHNIQUE}

The non-dominant pole $\left(f_{2}\right)$ and the zero $\left(f_{Z}\right)$ of a 2 stage miller-lead compensated opamp depend on $g m_{2 E}$ and $R_{C}$, respectively.

$$
f_{Z} \approx \frac{-1}{2 \pi R_{C} C_{C}} \quad f_{2}=\frac{-g m_{2 E}}{2 \pi\left(C_{C}+C_{L}\right)}
$$

If $R_{C}$ could be forced to track $1 / g m_{2 E}$ over process and temperature, then $f_{Z}$ would track $f_{2}$, thereby improving phase margin. Typically, this is accomplished through the use of stacked diode-connected MOSFETs as shown in Figure 1, where $V_{G S 2 B}=V_{G S 2 E}$, so that $V_{G S 4 C}=V_{G S 4 B}$. At steady state the effective resistance of $M_{4 C}$ in the triode region tracks $1 / \mathrm{gm}_{2 E}$. However, during transience $\left(I_{L} \neq 0\right)$, the gate-source voltages of the biasing transistors do not track $V_{G S 2 E}$. Shown in Figure 
6 is an alternative circuit that forces $R_{D S 4 C}$ to track $g m_{2 E}$ directly, even when $V_{G S 2 E} \neq V_{G S 2 B}$.

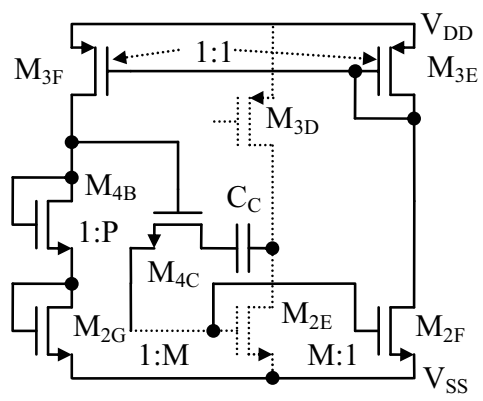

Figure 6: A new gm-tracking lead compensation resistor.

$M_{2 E}$ and $M_{3 D}$ once again form the $2^{\text {nd }}$ stage of an amplifier like the one in Figure 1. $M_{2 F}$ mirrors the current in $M_{2 E}$, and forces the same current through $M_{3 F}, M_{4 B}$, and $M_{2 G}$. Therefore $V_{G S 2 G}$ $=V_{G S 2 E}$, and $R_{D S 4 C}$ is given by (16), which is always independent of process, temperature, and load variations. Parameters $\mathrm{M}$ and $\mathrm{P}$ represent the transistor width ratios shown in Figure 6.

$$
R_{D S 4 C}=\frac{1}{g m_{2 E}}\left(\frac{M}{P}\right)
$$

To test the $g m$-tracking performance and stability of the circuit proposed in Figure 6, we designed three 2-stage opamps. All opamps have the architecture of the opamp in Figure 1 but use different lead compensation biasing techniques:

- Opamp A: passive $R_{C}$, as shown in Figure 1.

- Opamp B: MOSFET $R_{C}$, as shown in Figure 1.

- Opamp C: MOSFET $R_{C}$, as shown in Figure 6.

We tested the phase margin and stability of the opamps over load current variations by simulating them in open loop with current sinks attached to their outputs. Although opamps are rarely loaded with ideal current sources, the constant current source provides a convenient method of obtaining the frequency response under a variety of load conditions. We recorded the phase margin as we increased $I_{L}$ from $0 \mu \mathrm{A}$ to $110 \mu \mathrm{A}$, or $95 \%$ of the $2^{\text {nd }}$ stage bias current. As shown in Figure 7 the phase margin for opamp $\mathrm{C}$ (where $f_{Z}$ tracks $f_{2}$ ) is better under load than opamps A and B.

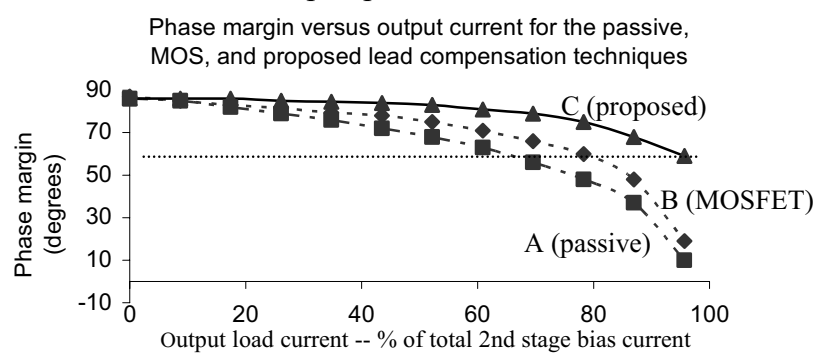

Figure 7: Phase margin vs. $\mathrm{I}_{\mathrm{LOAD}}$ for opamps A, B, and C.

To test the step response of the proposed circuit, we connected opamps $\mathrm{B}$ and $\mathrm{C}$ in non-inverting configuration with a closed loop gain of $6 \mathrm{~dB}$. We applied an input voltage pulse of $1.0 \mathrm{~V}$, and recorded the output voltages of the opamps, which are shown in Figure 8. Opamp B shows oscillations that arise because $M_{4 C}$ enters saturation. The oscillations are not present in the proposed circuit.

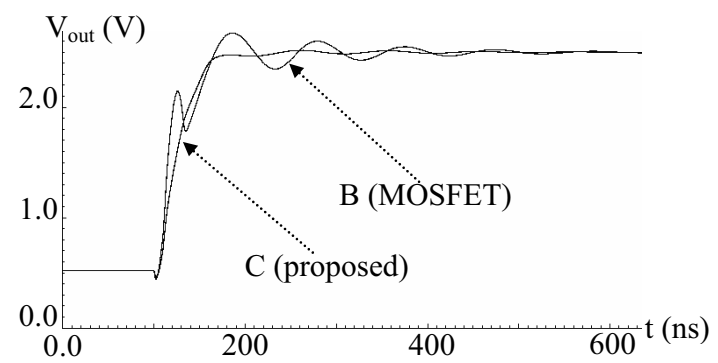

Figure 8: Output voltages for opamps B, and C during a step response simulation

Note that rising steps such as the one in Figure 6 can cause an opamp to slew if $M_{2 E}$ enters cutoff. In our proposed circuit in Figure $6, M_{2 F}$ and $M_{2 G}$ would also enter cutoff, causing $M_{4 C}$ to become an open circuit. In this situation, the opamp would lose compensation and become unstable, but only for the duration of the rising edge. This problem has two simple yet effective solutions: (1) A constant bias current much smaller than $I_{D 3 F}$ can be introduced in parallel with $M_{3 F}$, or (2) a diode connected MOSFET with high channel resistance (large $L$ ) can be added in parallel with $M_{4 C}$ or between $M_{4 B}$ and the gate of $M_{4 C}$.. The simulation results in Figure 8 were obtained after implementation of the first solution.

\section{CONCLUSION}

We have shown that, in general, the use of a constant gm bias circuit does not ensure constant transconductance throughout an integrated circuit, and have provided specific guidelines to minimize any variation. We have also demonstrated improved stability by adding a high impedance node to the constant-gm bias circuit. In fact, the same general technique and architectural modification can be applied to other current and voltage reference circuits. Finally, we have proposed a lead compensation biasing technique that improves opamp large signal settling time, and stability under load.

\section{REFERENCES}

[1] K. Martin, D.A. Johns, Analog integrated circuit design, New York: John Wiley \& Sons Inc., 1997.

[2] A. McLaren, and K. Martin, "Generation of Accurate OnChip Time Constants and Stable Transconductances," IEEE Journal of Solid-State Circuits, vol. 36, no. 4, April 2001.

[3] A.I.A. Cunha, M.C. Schneider, C. Galup-Montoro, "An MOS transistor model for analog circuit design," IEEE Journal of Solid-State Circuits, vol. 33, no. 10, Oct. 1998.

[4] J. M. Steininger, "Understanding wide-band MOS transistors," IEEE Circuits and Devices, vol. 6, pp. 26-36, May 1990.

[5] T. Pialis, "Design and Analysis Techniques for LowVoltage, Low-Jitter Voltage-Controlled Oscillators," M.Eng. Design Project Report, University of Toronto, Department of Electrical Engineering, Toronto, 2002. 